\title{
An EBSD Study of Gallium Arsenide Nanopillars
}

N. A. Bulloss , Joshua Shapiro ${ }^{* *}$, S.V. Prikhodko ${ }^{* * *}$, P.P. Camus

* Thermo Fisher Scientific, 5225 Verona Rd, Madison, WI 53711

UCLA Integrated Nanomaterials Laboratory University of California Los Angeles, Los Angeles, CA 90095

Department of Materials Science and Engineering, University of California Los Angeles, Los Angeles, CA 90095

GaAs semiconducting nanopillars are showing much promise in the field of solar cells, microelectronics and energy storage. For example, a surface composed of a carpet of light-absorbing nanopillars provides a large surface area and significantly improved light conversion efficiency compared to current silicon photovoltaic technology. GaAs nanopillars have the added advantage that they can be used to produce flexible solar cells.

In this study GaAs nanopillars grown under different conditions on GaAs (111) B (zincblende) substrate were analyzed in a field emission electron microscope using electron backscatter diffraction (EBSD). The nanopillars vary in size from $100 \mathrm{~nm}$ to $300 \mathrm{~nm}$ and vary in morphology from thin fibrous wires to larger cross-section columns. The nanopillars exhibit a wurtzite or zincblende type microstructure and the crystal structure can be directly related to the morphology of the GaAs rods. In the case of the longer nanopillars it is possible to sample multiple areas on the rods to examine crystallographic rotations during growth.

This study demonstrates the feasibility of using EBSD analysis in the SEM to characterize nanostructure materials. 